States; ${ }^{2}$ National Center for HIV, Viral Hepatitis, STD and TB Prevention, CDC, Atlanta, GA, United States; ${ }^{3}$ Safe Water and AIDS Project (SWAP), Kisumu, Kenya; ${ }^{4}$ Division of Foodborne, Waterborne and Environmental Diseases, CDC, Atlanta, GA, United States; ${ }^{5}$ Nell Hodgson Woodruff School of Nursing, Emory University, Atlanta, GA, United States; ${ }^{6}$ Center for Global Health, CDC, Atlanta, GA, United States

Objective Evaluate introduction of rapid syphilis tests (RSTs) into antenatal clinic (ANC) services at low-level health facilities in 2 rural districts in Nyanza Province, Kenya - assessing coverage, testing quality, treatment, data recording, and effect on HIV testing. Methods From March 2011 - February 2012, RSTs were introduced into ANC services at 25 rural facilities. Before introduction, handson training was conducted for nurses on use of RSTs, results counselling, appropriate maternal treatment, documenting data and proficiency testing. During the programme, 3 proficiency testing rounds were done. After the programme, ANC log-books from 8 priority clinics were used to assess data reporting and compare coverage of syphilis and HIV testing and syphilis treatment for the 12 -month intervals before and during the programme. Nurses and mothers were also interviewed.

Results Thirty-four nurses from 25 clinics were trained. Proficiency testing identified and corrected early RST problems. In the 8 priority clinics, syphilis testing at first ANC visit increased from $18 \%(279 / 1586$ attendees $)$ in the 12 months before to $70 \%$ $(1123 / 1614$ attendees) during the 12 -month programme $(p<0.001)$; 35 women $(3 \%)$ tested positive during the programme vs. $1(<1 \%)$ in the previous 12 months $(p<0.001)$. RST use and results were routinely documented, but no clinic recorded treatment per training. In 5 clinics, assessment of HIV test coverage was limited by lack of prior HIV-positivity data; however, records from 3 high-volume clinics suggested no difference in HIV testing rates before and during the programme. Interviews indicated many new nurses were not trained, while mothers reported limited counselling about testing or results.

Conclusions Introducing RSTs into rural ANC services greatly increased syphilis testing and detection without effects on HIV testing. We identified challenges in documenting treatment, counselling women appropriately, and adequate training. Amendments to existing and "refresher" training may improve services and documentation of treatment.

\section{P3.358 SYPHILIS SERO-POSITIVITY AMONGST PREGNANT WOMEN ATTENDING PUBLIC ANTENATAL CLINICS: A 5 YEAR (2004- 2008) ANALYSIS FROM 15 PUBLIC PRIMARY HEALTH CARE FACILITIES IN GABORONE, BOTSWANA}

doi:10.1136/sextrans-2013-051184.0811

'A B Ganiyu, 'L Mason. 'School of Medicine, University of Botswana, Gaborone, Botswana; 'University of Liverpool, Liverpool, UK

Background The objectives of study were to determine trends in syphilis prevalence, trends in the proportion screened and to compare with unscreened for syphilis amongst pregnant women attending 15 public antenatal clinics in Gaborone, 2004-2008.

Methods Descriptive quantitative study using routinely collected antenatal data.

Results The overall syphilis prevalence amongst pregnant women in Gaborone decreased from 2.96\% (95\% CI, 2.55-3.37) in 2004 to $1.15 \%$ (95\% CI, 0.89-1.41) in 2008 ( $p<0.001$ ), suggesting a significant downward trend in syphilis prevalence over a five years period. The age specific prevalence per total number of reactive VDRL/RPR was highest amongst pregnant women aged 26 to 30 years $(p<0.001)$ and lowest for those aged 16 to 20 years $(p<0.025)$ for 2004-2008, implying significant differences in the syphilis prevalence in each age group. However, the results reflect variations in syphilis prevalence rates within and between clinics.
There were slight fluctuations in the proportion of pregnant women screened for syphilis, ranged from $87.16 \%$ in 2004 to $91.20 \%$ in 2008. However, nearly all the clinics demonstrated no trends in the proportion of pregnant women screened for syphilis for 2004 2008.

Conclusion Syphilis sero-positivity in pregnancy in Gaborone has been declining for the last five years, but is far more prevalent amongst pregnant women aged 26 to 30 years and the lowest age specific prevalence was 16 to 20 years for 2004 to 2008 . This decline may be attributed to a number of factors and in particular, the adoption of syndromic approach for management of sexually transmitted infections in all clinics across the country. This study showed variations in the trend of syphilis prevalence by clinics and proportions of pregnant women screened for syphilis. However, a high proportion of pregnant women not screened for syphilis may have contributed to under-estimate syphilis prevalence rates.

\section{P3.359 MY GOODNESS! CONDOMS HAVE OTHER USES BESIDES USE DURING SEXUAL INTERCOURSE}

doi:10.1136/sextrans-2013-051184.0812

'T Agyarko-Poku, ${ }^{2} Y$ Adu Sarkodie, ${ }^{3} \mathrm{~L}$ Atakorah-Yeboah. 'Suntreso Government Hospital, Ghana Health Services, Kumasi, Ghana; ${ }^{2}$ School of Medical Sciences, Kwame Nkrumah University of Science and Technology, Kumasi, Ghana; ${ }^{3}$ Catholic University College, Fiapre, Sunyani, Ghana

Background Self-reported uses of condom have been documented in many studies as not good proxy indicator for their utilisation. Many players in health programmes in Ghana report high distribution of male and female condoms for HIV prevention programmes. This is not commensurate with their usage. Available recent study data prompted this study. The objective of this study is to determine if there are other uses for male and female condoms besides being use during sexual intercourse.

Methods Between January and October 2012, 987 (Male 48\% (474), Female 52\% (513) persons aged between 18 and 45 who consented to participate in this cross sectional study were recruited. A pretested structured questionnaire was administered to consenting individuals one-on-one in Western, Ashanti and Northern regions of Ghana. Sociodemographic characteristics. Data was analysed with SPSS 16.

Results $80 \%$ (790/987) reported that condom can be used to prevent Urinary Tract Infections by wearing it during swimming in ponds and rivers.74\% (730/987) reported that condoms are used to set fires especially for commercial use. The rubber and oil in the condom support fire setting quicker than using paper especially when charcoal and firewood is used as a cooking fuel.68.0\% (671/987) reported that bangles and earrings could be made out of female condoms for sale.

Conclusion Condoms have other uses which has both commercial and social potential to compete favourably with the traditional use of condom for sexual intercourse to provide dual protection i.e. prevention of sexually transmitted infections including HIV and unwanted pregnancy. This study presents evidence that programmes need to use several methods to validate use of condoms for their intended purpose as data on condoms distributed could mislead the indicators on unmet needs for contraceptive and Couple Years of Protection (CYP).

\section{P3.360 AN ASSESSMENT OF THE KNOWLEDGE AND PRACTICAL SKILLS IN USING MALE CONDOMS AMONG THE GENERAL PUBLIC}

doi:10.1136/sextrans-2013-051184.0813

S L Kay, K Radcliffe. Whittall Street Clinic, Birmingham, UK 